\title{
DYSVERSION OF LATERAL EYELASHES IN CHILDREN: A NEW DIAGNOSIS
}

\author{
S. A. SADIQ and R. N. DOWNES \\ Nottingham
}

\begin{abstract}
SUMMARY
We believe that a previously undocumented cause of recurrent red, watery, sore and itchy eyes in children is dysversion of the lateral eyelashes. Diagnosis requires a close examination of the lateral aspect of the eyelids where dysverted lashes are seen to be growing from normal follicles. The lateral lashes are fine, long and flexible. They curve inwards and rub on the conjunctiva and cornea, and cause the above symptoms. Secondary signs include skin changes, recurrent conjunctivitis, and corneal abrasions. Conservative treatment consisting of manual repositioning of the dysverted eyelashes is recommended so that surgery may be avoided. Recognition of the condition by the primary practitioner, and institution of the straightforward treatment described above, can obviate much morbidity. The inappropriate use of topical antibiotics and frequent return visits to the practitioner may also be thus avoided.
\end{abstract}

Recurrent red, sticky and watery eyes are common presenting symptoms in children who attend a primary health care practitioner. We describe a condition whereby dysversion of the lateral eyelid lashes may represent a specific cause of these common symptoms, and one which has not previously been documented. Diagnosis requires a thorough history and examination, with particular attention to the lateral aspects of the eyelids. These dysverted lashes are of normal morphology and arise from normal follicles. They are fine, long and abnormally shaped, and thus may abrade the conjunctiva and cornea.

The case reports of four children with this condition are presented.

\section{Case 1}

\section{CASE REPORTS}

A 3-year-old boy was referred for nasolacrimal system probing. He had a history of recurrent red,

Correspondence to: Mr R. N. Downes, Consultant Ophthalmologist, Queen's Medical Centre, University Hospital, Nottingham NG7 2UH, UK. sticky and watery eyes since birth. More recently, there had been irritation of both eyes, with lateral skin excoriation. He had previously been treated with topical antibiotics by his general practitioner, but with no improvement. The patient was otherwise fit and well. There was no personal or family history of allergic or systemic disease.

On examination, the vision was equal in both eyes with no refractive error. The child was noted to have dysversion of the lateral lashes of the upper eyelids bilaterally. The lateral lashes were long and fine, and became trapped under the lateral canthal regions between the two conjunctival surfaces. There was localised crusting and debris on the eyelashes. The skin lateral to the eyelids was dry and excoriated. Otherwise the eyelids were in a normal position and no ocular abnormality was found.

Treatment consisting of manual repositioning of the dysverted eyelashes was advised. This led to rapid and complete resolution of symptoms. A short time after stopping the treatment, the parents reported a return of lateral eyelash misdirection with a recurrence of symptoms. Again repositioning resulted in a prompt resolution, since when there has been no recurrence.

\section{Case 2}

A 3-year-old boy attended his general practitioner in January 1993 after his parents had noticed that the child was blinking excessively. The patient also complained that his eyes hurt. The child was otherwise healthy with no systemic symptoms. The general practitioner noticed some ingrowing eyelashes at the lateral sides of both upper eyelids. The offending lashes were epilated successfully on the right, but this was not possible on the left. Epilation resulted in a resolution of symptoms for 6 months. The patient returned to the general practitioner in June 1993 with a recurrence of blinking and irritation due to ingrowing lashes in both upper eyelids.

Specialist examination revealed the patient to have 


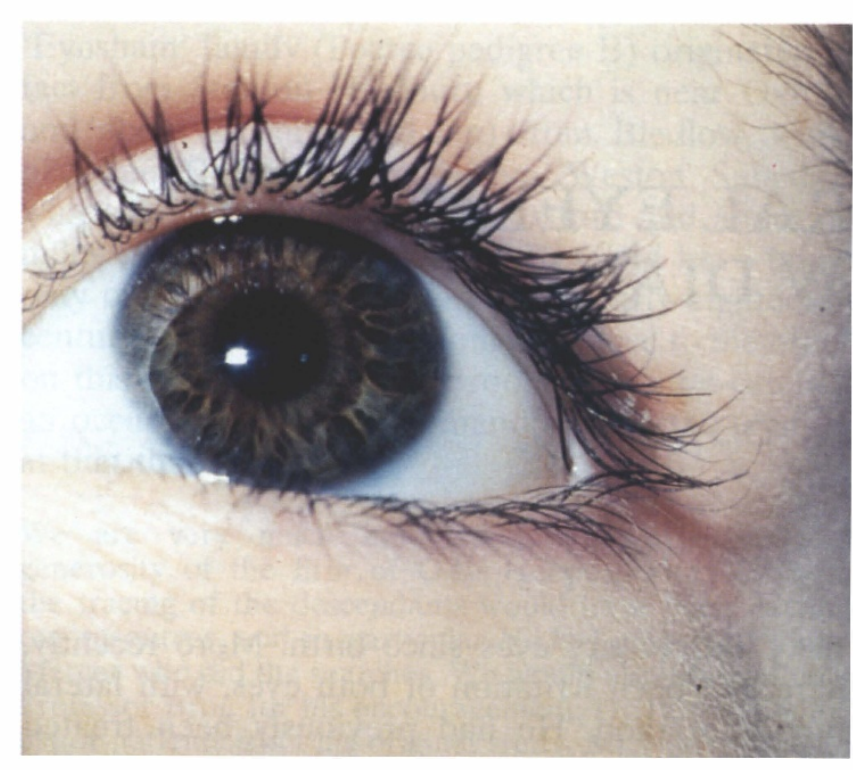

Fig. 1 Case 1. Several matted lashes are shown trapped under the upper lid margin. Lateral skin excoriation is also present.

equal vision in both eyes with no refractive error. It was noticed that the lateral eyelashes arose from the correct position but were quite flexible and turned back on themselves to become trapped on the under surface of the eyelids laterally. Otherwise the eyelid positions were normal, and no other ocular abnormality was detected. The patient's mother was advised on the appropriate treatment, which led to resolution of the child's symptoms within 1 month. There was a recurrence of symptoms 4 months later once the manual repositioning had been stopped. These rapidly resolved on reinstituting treatment, since when there has been no recurrence.

\section{Case 3}

A 4-year-old general practitioner's son was referred to the eye clinic with a history of recurrent red and sticky eyes. The child was prone to rubbing his eyes excessively. There had been a variable response to treatment with a variety of topical antibiotics. The patient was otherwise healthy and there was no other history of note.

The child had equal vision in both eyes and no refractive error. On examination, bilateral misdirected lashes were seen arising from the lateral aspects of the upper eyelids. The lashes were long and turned underneath the lid margins. The eyelids and eyes were otherwise normal. Appropriate treatment was instigated with prompt resolution of symptoms without any recurrence.

\section{Case 4}

A 5-year-old Indian boy was initially seen by an optician with a history of sore and itchy eyes. The patient did not suffer from any allergic conditions and was in good general health. There were no familial ocular or general problems. The eyes were not red or sticky. The optician's notes at that time reported a mild bilateral lower lid entropion, but no referral was made to the general practitioner.

The child was seen by an ophthalmologist at the age of 7 years with blurry vision and sore irritable eyes. His mother reported that he often rubbed his eyes. Several dysverted lashes were noted at the lateral margins of both upper eyelids. The eyelids were not malpositioned. There were no signs of epiblepharon, trichiasis or entropion. No corneal or other ocular pathology was seen. His mother was advised of the appropriate treatment, which led to resolution of symptoms without any recurrence.

\section{DISCUSSION}

Sore, irritable, itchy or discharging eyes are common complaints in children. These children initially present to, and are treated by, their general practitioner. There are many causes for these symptoms, but we believe dysverted eyelashes to be a newly described cause. It is an easy condition to overlook or misdiagnose.

These dysverted eyelashes are normal cilia arising from normal follicles, and usually arise from the extreme lateral margin of the upper eyelid. The cilia are fine and long, and hence flexible. An initiating factor, perhaps the tangential direction of growth, allows these lateral lashes to become trapped underneath the eyelid margins or lateral canthal tendons. Wetting and softening of the lashes causes them to become misshaped or dysverted, so that they come to lie between the tarsal and bulbar conjunctival surfaces. We postulate that this sequence of events leads to the symptoms described above, and a continuation of the cycle of symptoms and problems. The number of lashes affected may number from one to several.

The diagnosis depends on the practitioner being aware of this condition, and taking care to examine the most lateral parts of the eyelids. The dysverted eyelashes may be seen to rub on the lateral conjunctiva in primary gaze position, and may abrade the cornea on lateral gaze. This leads to the patient suffering the symptoms described above. Secondary features may include skin excoriation, recurrent conjunctivitis and corneal abrasions.

The dysverted lashes seen in our patients need to be differentiated from several other conditions. Trichiasis is a misalignment of the lashes due to scarring of the follicles. In lower lid entropion, the entire eyelid margin turns inwards, becoming outwards when the lower lid is pulled down. Cicatricial upper eyelid entropion may be laterally based, but is usually accompanied by other signs. Epiblepharon affects the medial portion of the eyelids, and is characterised by an extra fold of eyelid skin which 
stretches across the upper or lower eyelid. Epiblepharon with inverted lashes is often found in Japanese children and tends to disappear spontaneously with age. ${ }^{1}$ Most cases $(78 \%)$ of epiblepharon produce no symptoms, despite corneal touch. This may be explained by the fineness of the lashes and the tolerance of the cornea. For symptomatic patients, eyelid everting sutures may be placed in the first instance, to be followed by either buried sutures or skin resection operations in those in whom there is a recurrence.

We recommend conservative treatment for the lateral lash dysversion syndrome. Treatment involves recognition of this condition, followed by manual repositioning of the affected eyelashes in an external direction. The parents are asked to observe the lateral aspects of the upper eyelids, and then reposition the dysverted eyelashes with a moistened cotton-tipped applicator. This is initially performed twice daily, and then reduced to once daily, as the dysverted eyelashes take up an outward curving shape. The patient is encouraged not to rub the eyes as the rubbing action may also prevent or delay reshaping of the eyelash curvature. When the patient is asymptomatic, and all the lashes are seen to be of the same outward curvature, manual reshaping can be discontinued on a regular basis, but the parents are asked to continue observing the shape of the lateral lashes, and to perform repositioning as and when required. Treatment may need to be reinstituted if there is a recurrence of symptoms, or if dysversion of the eyelash shape is seen again.

Epilation of eyelashes, or surgery in the form of eyelid everting sutures, is not advocated as the condition settles extremely well with reshaping of the lashes alone. In this way, the child avoids the discomfort and inconvenience of surgery, and also the symmetry of the eyelids is maintained.

Many questions remain unanswered regarding the lateral lash dysversion syndrome. What controls normal eyelash length and thickness? Why are only young boys affected in our series of patients? Does the condition also occur in adults? Is the condition always bilateral? What initiates the condition? The normal pattern of eyelash growth shows finer and shorter lashes to be present medially and laterally. In our four patients, there seems to be no significant morphological difference in the appearance of the eyelashes, except that the lateral lashes are longer than normal - as long as the central lashes. We postulate that the length of the lashes, combined with their fineness, may render them more flexible. An initiating factor, perhaps their tangential direction, allows these longer and more flexible lashes to become trapped behind the lateral canthal tendon, and causes their reshaping or dysversion into an inward direction. The length and fineness of the lashes also seems to contribute to difficulty in their removal from the lateral canthal area. Presumably, it is this same condition which caused the early symptoms in our patients.

Key words: Eyelid, Eyelash, Dysversion, Children, Conservative treatment.

\section{REFERENCE}

1. Noda S, Hayasaka S, Setogawa T. Epiblepharon with inverted eyelashes in Japanese children. I. Incidence and symptoms. Br J Ophthalmol 1989;73:126-7. 